\title{
Materials Development Aided by Atomic-Resolution Electron Microscopy
}

\author{
Randi Holmestad ${ }^{1}$, Sigurd Wenner ${ }^{1}$, Magnus Nord ${ }^{1}$, Per Erik Vullum ${ }^{1,2}$ and Calin Marioara ${ }^{2}$ \\ ${ }^{1}$ Department of Physics, Norwegian University of Science and Technology (NTNU), 7491 Trondheim, \\ Norway \\ ${ }^{2}$ SINTEF Materials and Chemistry, 7034 Trondheim, Norway
}

In the end of 2013 the TEM Gemini Centre at NTNU and SINTEF, Trondheim, Norway, installed a double corrected JEOL ARM200CF equipped with a large angle EDS detector and a GIF Quantum with Dual-EELS capabilities as a part of the NORTEM investment [1]. This presentation will focus on how we use the high angle annular dark field scanning transmission electron microscopy (HAADF-STEM) technique to get useful information at the atomic scale from different materials. The talk will concentrate on Al alloys as it is an important research activity in the Gemini Centre and supported by Norwegian Aluminum industry. Also a few other examples from functional materials will be shown.

Age hardenable aluminum alloys such as 2xxx, 6xxx and 7xxx alloys are important as structural materials for automotive and aerospace applications due to properties like high strength/weight ratio, good formability and weldability, often combined with good corrosion resistance. The alloys acquire strength through the formation of nanoscale, metastable precipitate phases during heat treatment. Our objective is to understand more of the fundamental physics going on at the atomic scale, which governs nucleation, phase stabilization and in general precipitation in these alloys. Strengthening properties of age-hardening precipitates depend on their atomic structure, morphology and coherency with the $\mathrm{Al}$ matrix, which is influenced by the alloy composition and the thermo-mechanical history of the material. Therefore, studying precipitates in detail is fundamental in alloy development. Some elements affect the precipitation significantly at low concentration levels, e.g. 0.01\%. In recycled Al alloys several such impurity elements can be both beneficial and detrimental to the properties. Through micro-alloying, certain elements can also be added on purpose to improve material properties. The main hardening precipitates in Al-Mg-Si (6xxx) alloys form and grow as needles along <100>Al, and are observed in cross-section when the material is tilted to a $<100>\mathrm{Al}$ zone axis. HAADF-STEM is an excellent technique for studying the distribution of heavier elements such as $\mathrm{Ag}, \mathrm{Cu}$ and $\mathrm{Zn}$ in the precipitates [2]. Figure 1 illustrates how aberration corrected STEM gives both high resolution and compositional information of a Q' type precipitate containing $\mathrm{Cu}$. Comparing a high resolution, an uncorrected and a corrected HAADF-STEM image, we see that in the latter every atomic column in the precipitate is resolved, and the distribution of the $\mathrm{Cu}$-containing columns is clear. When $\mathrm{Cu}, \mathrm{Zn}$ or $\mathrm{Ag}$ is added to 6xxx alloys, the precipitate structures often become disordered. Although the disordered precipitates lack periodicity in their cross-sectional planes, they all contain an ordered network of Si atomic columns having a projected hexagonal symmetry. The disordered precipitates consist of fragments of known phases in the Al-Mg-Si(-Cu) alloy system connected through the common Si-network [3].

Other examples are from studies of interfaces in perovskite materials. HAADF-STEM is used to ensure the quality and measure the strain in the epitaxial interface between $\mathrm{La}_{0.7} \mathrm{Sr}_{0.3} \mathrm{MnO}_{3}$ (LSMO) thin films and $\mathrm{SrTiO}_{3}$ (STO) substrates [4]. Lead free piezoelectric materials are a challenge. A very simple spin coating technique can be used to synthesize piezoelectric $\mathrm{K}_{0.5} \mathrm{Na}_{0.5} \mathrm{NbO}_{3}(\mathrm{KNN})$ films on top of STO with various substrate orientations (i.e. (001), (011) and (111) STO wafer orientations). The interface between the film and the substrate is one important parameter for the functional properties of the film. 
Here we demonstrate how HAADF-STEM can be used to study the interface structure, as shown in figure 2. The presentation will show how advanced TEM methods, and in particular aberration probe corrected HAADF-STEM are used to acquire useful atomic information from our samples [5].

\section{References:}

[1] http://www.nortem.no/

[2] T Saito, CD Marioara, SJ Andersen, W Lefebvre and R Holmestad, Phil. Mag., 94 (2014) 520.

[3] FJH Ehlers, S Wenner, SJ Andersen, CD Marioara, W Lefebvre, C Boothroyd, R Holmestad, J. Mater. Sci. 49 (2014), 6413.

[4] M Nord, PE Vullum, M Moreau, JE Boschker, SM Selbach, R Holmestad, and T Tybell, Appl. Phys. Lett. 106 (2015) 041604.

[5] The TEM work was carried out on the NORTEM facility. The authors acknowledge Profs. M-A Einarsrud from Materials Science and Engineering and T Tybell from Electrical Engineering, both at NTNU, for providing the perovskite materials.
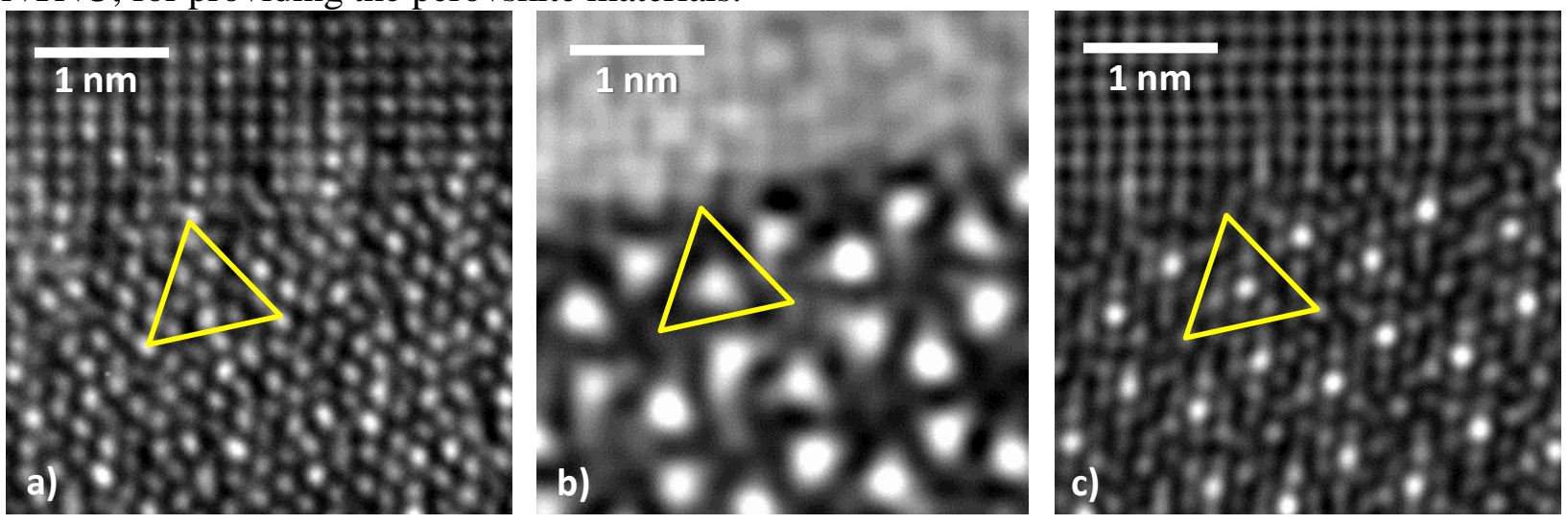

Figure 1. Example of a) HRTEM (JEOL2010F), b) uncorrected HAADF-STEM (JEOL2010F) and c) aberration corrected HAADF-STEM (JEOL ARM200CF) techniques used to image the Q'-phase precipitate structure in an $\mathrm{Al}-\mathrm{Mg}-\mathrm{Si}-\mathrm{Cu}$ alloy.
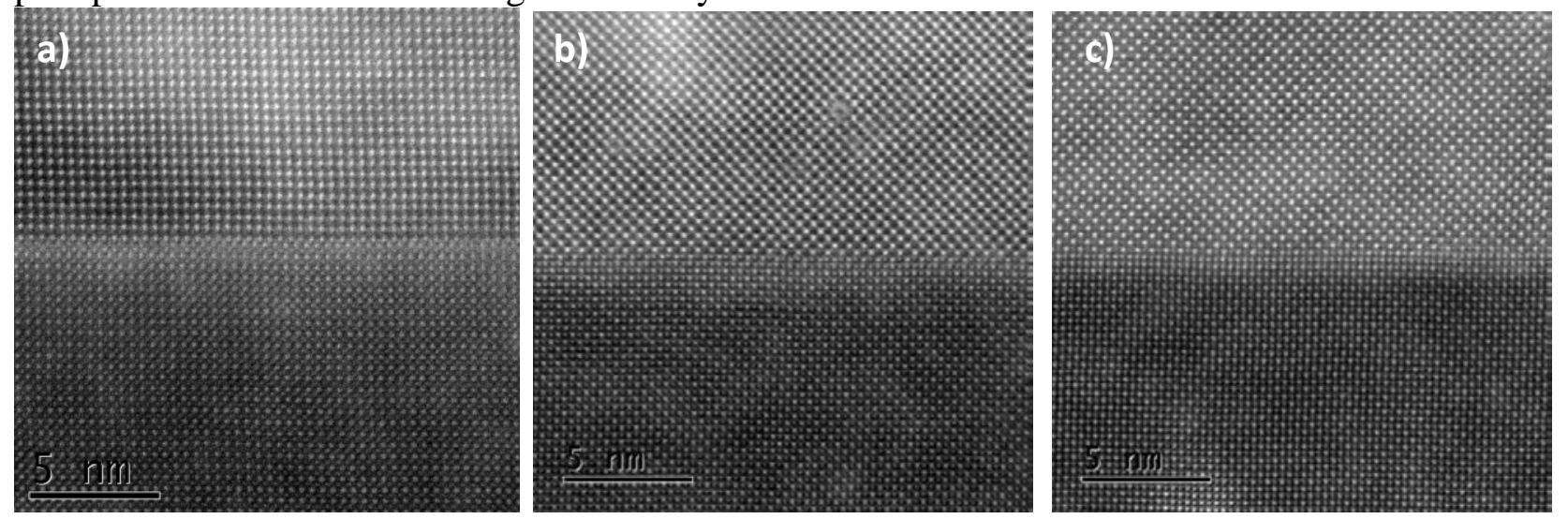

Figure 2. HAADF-STEM images of the interface between STO and KNN. (a) The KNN film grows epitaxially on top of the (001)STO substrate. It can be seen that the STO substrate terminates with a SrO layer and the KNN film starts with a $\mathrm{NbO}_{2}$ layer. In (b) and (c) the KNN film is grown on top of (011)STO. The difference between (b) and (c), except that they are acquired at slightly different interface locations, is the dark field STEM collection angles. In (b) the inner and outer angles are 43 and 170 mrad, while in (c) they are reduced to cover the range $37-146$ mrad. The decrease in collection angles makes the light weight $\mathrm{A}$ cations $(\mathrm{K}$ and $\mathrm{Na}$ ) visible as very weak bright dots. 